\title{
Use of confocal laser endomicroscopy with a fluorescently labeled fatty acid to diagnose colorectal neoplasms
}

\author{
Feihong Deng ${ }^{1, *}$, Yuan Fang ${ }^{1, *}$, Zhiyong Shen ${ }^{2}$, Wei Gong ${ }^{1}$, Tao Liu' ${ }^{1}$, Jing Wen ${ }^{1}$, Wanling \\ Zhang ${ }^{4}$, Xianjun Zhu ${ }^{2}$, Hui Zhong ${ }^{1}$, Tong Wang ${ }^{4}$, Fachao Zhi ${ }^{1}$ and Biao $\mathrm{Nie}^{3}$ \\ ${ }^{1}$ Department of Gastroenterology, Nanfang Hospital, Southern Medical University, Guangzhou, Guangdong Province, 510515 \\ China \\ ${ }^{2}$ Department of General Surgery, Nanfang Hospital, Southern Medical University, Guangzhou, Guangdong Province, 510515 \\ China \\ ${ }^{3}$ Department of Gastroenterology, First Affiliated Hospital of Jinan University, Jinan University, Guangzhou, Guangdong \\ Province, 510630 China \\ ${ }^{4}$ Key Laboratory of Functional Protein Research of Guangdong Higher Education Institutes, Institute of Life and Health \\ Engineering, College of Life Science and Technology, Jinan University, Guangzhou, Guangdong Province, 510632 China \\ *These authors contributed equally to this work
}

Correspondence to: Biao Nie, email: niebiao2@163.com

Keywords: fatty acid metabolism, colorectal neoplasm, confocal laser endomicroscopy, fluorescent agents, de novo FA synthesis

Received: April 19, 2017

Accepted: July 11, 2017

Published: July 24, 2017

Copyright: Deng et al. This is an open-access article distributed under the terms of the Creative Commons Attribution License 3.0 (CC BY 3.0), which permits unrestricted use, distribution, and reproduction in any medium, provided the original author and source are credited.

\section{ABSTRACT}

Endoscopic treatment for early colorectal cancer closely correlates with patient prognosis. However, endoscopic differentiation between carcinomas and nonneoplastic lesions remains difficult. Here, we topically stained colorectal neoplasms with a fatty acid analogue (BODIPY-FA) and quantified the fluorescent signals using confocal laser endomicroscopy (CLE) and fluorescence microscopy. We also analyzed protein expression in colorectal cancer tissues. We found that expression of fatty acid synthase was elevated, while the expression of fatty acid transporters was reduced in colorectal cancer. In colorectal cancer mouse models and patients, the BODIPY-FA signals were higher in normal epithelia than in carcinomas or colonic intraepithelial neoplasias. BODIPY-FA staining revealed both the arrangement of intestinal glands and the intracellular structures under CLE screening. In a double-blind trial, CLE images stained with BODIPY-FA exhibited greater consistency $(\kappa=0.68)$ and overall validity $(74.65 \%)$ than those stained using intravenous fluorescein sodium $(K=0.43$, $55.88 \%$ ) when the results were compared with histological diagnoses. These findings suggest that topical use of BODIPY-FA with CLE is a promising imaging approach for early colorectal neoplasm screening.

\section{INTRODUCTION}

Cancer cell metabolism differs significantly from normal cell metabolism. Most proliferating cancer cells take up more glucose and produce more lactate via the glycolytic pathway than normal cells, even when oxygen is abundant [1-5]. This so-called 'Warburg effect' has been intensively studied and generally applied in clinical positron emission tomography-computed tomography $[6,7]$. In addition, endogenous fatty acid (FA) synthesis (also called de novo FA synthesis) is specifically enhanced in cancer cells, irrespective of the exogenous lipid supply [8-11]. This process is another important metabolic hallmark of cancer [12-14]. De novo FA synthesis generates long-chain fatty acids (LCFAs) from acetyl-CoA, and accounts for the synthesis of almost all esterified FAs and more than $93 \%$ of triglycerides to meet the biosynthetic demands of tumors [15]. However, the practical applications of this phenomenon have not been fully explored. 
As colorectal cancer (CRC) is the third-most-common cancer in males and the second-most-common cancer in females worldwide [16], early CRC detection is crucial for improving the quality of life and the cure rate for patients. Colonoscopy has been regarded as the gold standard for screening people at high risk for CRC. Multiple novel endoscopic technologies have recently been investigated, including narrow-band imaging (NBI), Fujinon intelligent chromoendoscopy (FICE), magnifying chromoendoscopy (MCE) and confocal laser endomicroscopy (CLE) $[17,18]$. CLE integrates experimental confocal microscopy with conventional optical endoscopy, magnifying regions of interest (ROIs) 1000-fold at the cellular and sub-cellular levels and allowing real-time in vivo histology in the clinical diagnosis of colorectal neoplasms [19, 20]. Although fluorescently labeled antibodies and peptides have been tested with CLE [21-24], labeled FAs have never been used in trials to evaluate colorectal neoplasms specifically on a metabolic level.

The fluorescently labeled FA analogue BODIPY-FA (4,4-difluoro-5-methyl-4-bora-3a,4a diaza-s-indacene-3dodecanoic acid) is activated by argon-ion laser excitation at $488 \mathrm{~nm}$, and has generally been applied for experimental research in lipid trafficking [25]. In this study, we found that BODIPY-FA was a promising biomarker for tumor imaging, due to the reprogramming of FA metabolism in CRC cells. Furthermore, upon its absorption, BODIPY-FA was enriched in the cytoplasm, and created contrast that allowed negative nuclear visualization. Thus, BODIPY-FA provided histological imaging similar to hematoxylin and eosin (H\&E) staining, making it superior to other newly developed contrast agents.

\section{RESULTS}

\section{FA metabolism differed between normal colorectal and CRC samples}

Analysis of data from the Clinical Proteomic Tumor Analysis Consortium indicated that most enzymes involved in de novo FA synthesis, including FASN, acetylCoA carboxylase (ACC) and ATP citrate lyase (ACLY), were expressed at significantly higher levels in CRC tissues than in normal colorectal tissues. In addition, the acetylCoA synthetase complex (ACSc) and carnitine palmitoyl transferase 2 (CPT2) were downregulated in CRC tissues, suggesting that FA oxidation is impaired in colonic carcinoma (Figure 1A and Supplementary Figure 1, detailed in Supplementary Table 4). More importantly, concomitant with the enhancement of endogenous FA synthesis, the expression of membranal transporters of LCFAs, such as plasma membrane FABP (FABPpm) and FABP1, decreased sharply in CRC (Figure 1A and Supplementary Figure 1). Western blotting revealed that FASN protein expression was elevated in CRC tissues, while real-time PCR and Western blotting indicated that
FABP1, CD36 and Caveolin-1 mRNA and protein levels were reduced in CRC (Figure 1B, 1C), potentially due to the reduced availability of exogenous lipids for the tumor.

LCFA transport begins with the uptake of circulating non-esterified FAs (derived from triglycerides) by membranal transporters. Previously, FA uptake studies relied on radiolabeled lipids, and thus lacked real-time dynamic monitoring and were costly [32]. BODIPY-FA is a fluorescently labeled FA consisting of a 12-carbon saturated FA chain connected to the fluorophore BODIPY. BODIPY-FA biologically resembles an 18-carbon FA, and is reported to be a suitable alternative to radiolabeled FAs [33]. Stearic acid (C18:0) is a dominant member of the non-esterified LCFA family, and is the main source of FA oxidation for cellular energy after its uptake by lipid transporters [34]. Through FACS analysis, we found that stearic acid could effectively compete with the BODIPYFA signal in SW480 cells (Figure 1D), confirming that BODIPY-FA is a C18:0 analogue. Thus, BODIPY-FA could be a tool for monitoring lipid transport, and could reflect the natural uptake of circulating non-esterified LCFAs in vivo.

\section{BODIPY-FA uptake was reduced in human CRC cells}

Based on the downregulation of FA transporters in human CRC tissues, we hypothesized that exogenous FA uptake is limited in cancer. To verify this hypothesis, we used BODIPY-FA to examine the differences in FA metabolism between CRC cells and normal colonic cells in vitro. FACS analysis demonstrated that BODIPY-FA accumulated in adherent cells within 15 minutes, and that the fluorescent signal was greater in a normal colonic cell line (FHC) than in CRC cell lines (SW480, HCT116, and LOVO) $(P<0.0001$, Figure 2A). FACS analysis of three pairs of fresh cancerous and matched normal colonic tissues revealed the same trend in the BODIPY-FA signal, and the absorption of normal primary colonic epithelial cells plateaued within five minutes (Figure 2B).

Next, we determined the ability of confocal laser microscopy to locate and discriminate BODIPY-FA staining. A stronger cellular signal was observed for FHC cells than for CRC cells (SW480, LOVO) $(P<0.0001$, Figure 2C). In addition, the specific BODIPY-FA signal was cytoplasmic and perinuclear (Figure 2D-2F). These data indicated that LCFA uptake was reduced in CRC cells, and that limited exogenous FA usage may be an intrinsic signature of tumor lipid metabolism.

\section{Fluorescence microscopy revealed reduced BODIPY-FA uptake by colonic neoplasms in mouse models in vivo}

Next, we topically administered fluorescent agents to both AOM/DSS-induced and xenograft models, and 
analyzed the cytoplasmic fluorescence intensity under fluorescence microscopy. When we analyzed the colitisrelated carcinomas produced in the AOM/DSS model, we found a stepwise decrease in the BODIPY-FA signal, which was strong in non-neoplastic samples, lower in CIN samples, and even lower in adenocarcinoma samples (Figure 3A, 3B, Supplementary Figure 3A). In addition, the BODIPY-FA signals from adenocarcinoma and CIN samples were approximately $39.3 \%(P<0.0001)$ and $17.7 \%(P=0.0117)$ lower, respectively, than in normal epithelia (Figure 3D). The BODIPY-FA signals from orthotopically implanted tumors of xenograft mice were approximately $10.2 \%$ lower than those of paired normal mucosae $(P=0.0017$, Figure $3 \mathrm{C}, 3 \mathrm{E})$.

Based on this difference, we used the deoxyglucose analogue 2-NBDG as a control, in which 2-deoxyglucose is linked to the fluorophore $\mathrm{N}$-(7-nitrobenz-2-oxa-1,3diaxol-4-yl)amino (NBD). The 2-NBDG signals were approximately $20 \%$ greater in neoplastic mucosae than in normal mucosae from AOM/DSS-induced mice $(P=0.0119$, Supplementary Figure 2A, 2C). The 2-NBDG absorption was also higher in inflamed tissues than in normal mucosae $(P=0.0209$, Supplementary Figure 3B). However, there were no differences between the normal tissues and the tumors in the xenograft model after 2-NBDG staining (Supplementary Figure 2B). All fluorescent agent signals were greater than the background autofluorescence.

\section{CLE revealed reduced BODIPY-FA uptake by colonic neoplasms in mouse models in vivo}

Because of the BODIPY-FA signal differences observed with fluorescence microscopy, we then performed CLE with topically applied fluorescent agents to explore the uptake differences in vivo. In AOM/ DSS-induced mice, the BODIPY-FA signals declined progressively from inflamed to normal to $\mathrm{CIN}$ to adenocarcinoma tissues (Figure 4A, 4B and Figure 5A). The mean greyscale values of the normal mucosae were 2.5 and 1.7 times greater than those of adenocarcinomas and CIN, respectively (Figure 4C). In parallel, the BODIPY-FA signal was 2.1-fold higher in normal tissues than in colonic orthotopic tumors (Figure 5C, 5D). For $\mathrm{APC}^{-\mathrm{min}}$ mice, the BOPIDY-C12 signal was significantly higher in normal mucosae than in adenomas, according to $\operatorname{CLE}(P<0.0001)$ and fluorescence microscopy $(P=0.0001)$ (Figure 4D-4F). Furthermore, changes
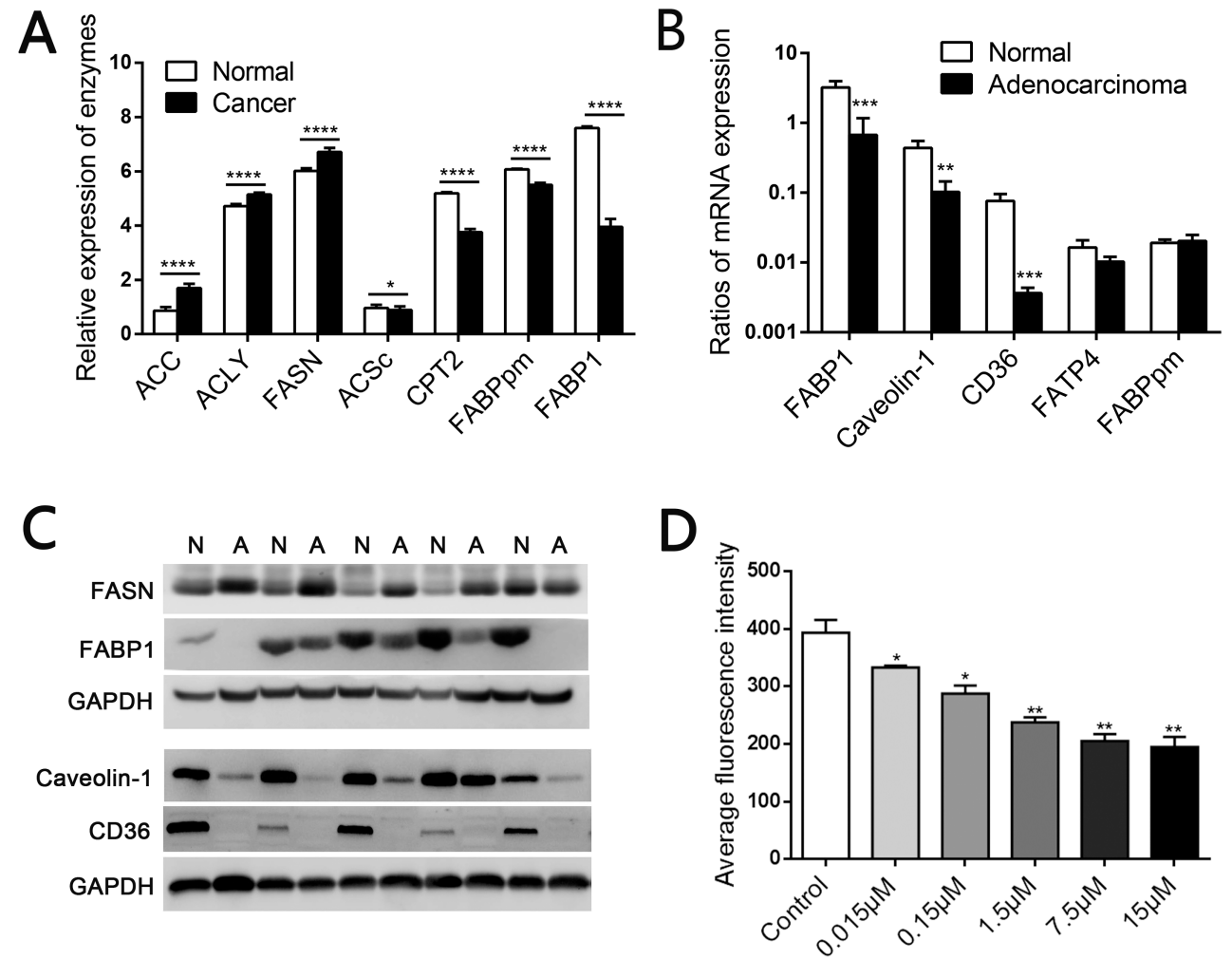

Figure 1: Elevated de novo fatty acid synthesis combined with reduced fatty acid oxidation in human CRC. (A) Mass spectrometry-based proteomic analysis of 95 colorectal cancer samples from the Clinical Proteomic Tumor Analysis Consortium. ACC, acetyl-CoA carboxylase; ACLY, ATP citrate lyase; FASN, fatty acid synthase; ACSc, acetyl-CoA synthetase complex; CPT2, carnitine palmitoyl transferase 2. (B) The mRNA levels of relevant LCFA transporters including FABP1, Caveolin-1 and CD36 were lower in CRC tissues than in matched normal mucosae. (C) The protein levels of FASN, FABP1, Caveolin-1 and CD36 in CRC tissues and matched normal mucosae. N, Normal; A, Adenocarcinoma. (D) Competition in the fluorescent signal after various concentrations of C18:0 were added to $2 \mu \mathrm{M}$ BODIPY-FA medium on SW480 cells. ${ }^{*} P<0.01, * * P<0.001, * * * P<0.001, * * * * P<0.0001$. Bars indicate the SEMs. 
in cellular structures, such as the disorganized cellular arrangement and cellular atypia in the neoplastic lesions, were more well-defined under CLE with BODIPY-FA staining than with other contrast agents.

In contrast, CLE following topical administration of 2-NBDG did not facilitate clear outlining of the glands, let alone cytoplasmic imaging, and no fluorescent difference between neoplastic and non-neoplastic tissues was observed in the mouse models (Figure 5B, 5E). For acriflavine staining, the nuclei within the ROIs were all displayed (Supplementary Figure 4A, 4B). No background autofluorescence was observed in normal or malignant tissues.

\section{CLE revealed reduced BODIPY-FA uptake in colonic neoplasms in patients}

We further used BODIPY-FA with CLE to explore lipid uptake in human samples. The mean greyscale values of all distinguishable confocal images (149 non-neoplasm
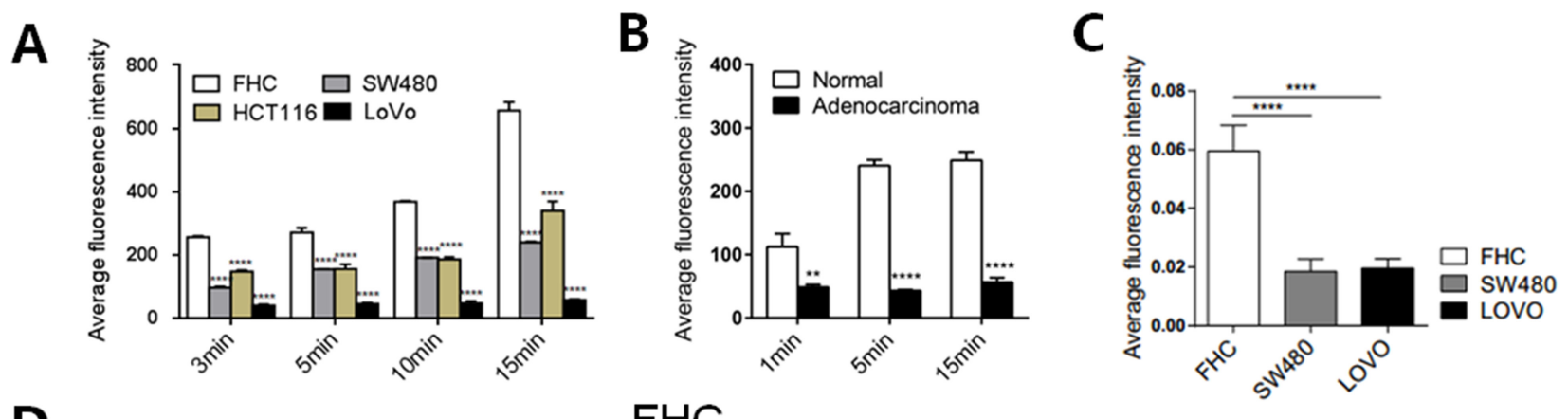

D

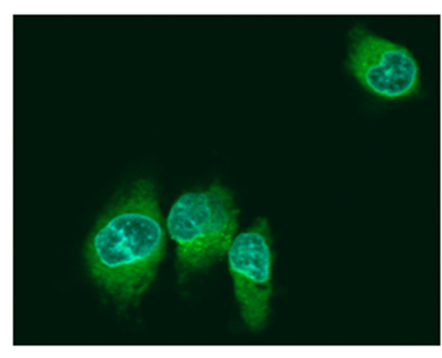

$\mathrm{FHC}$
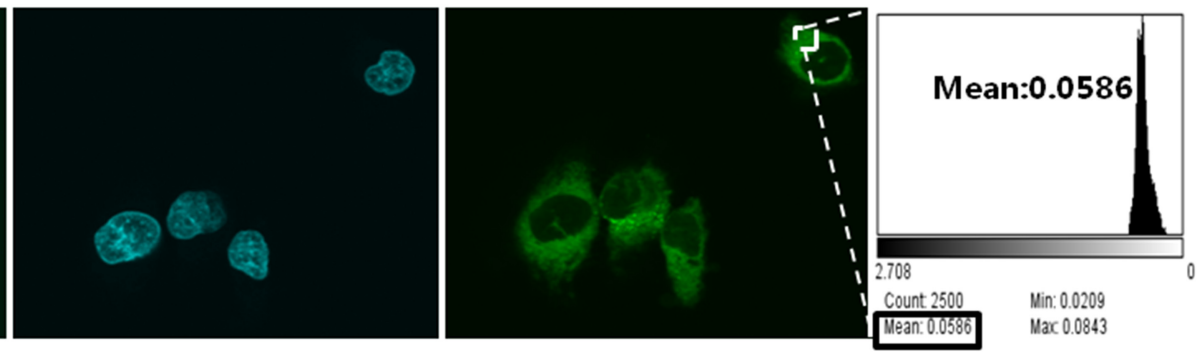

E

SW480
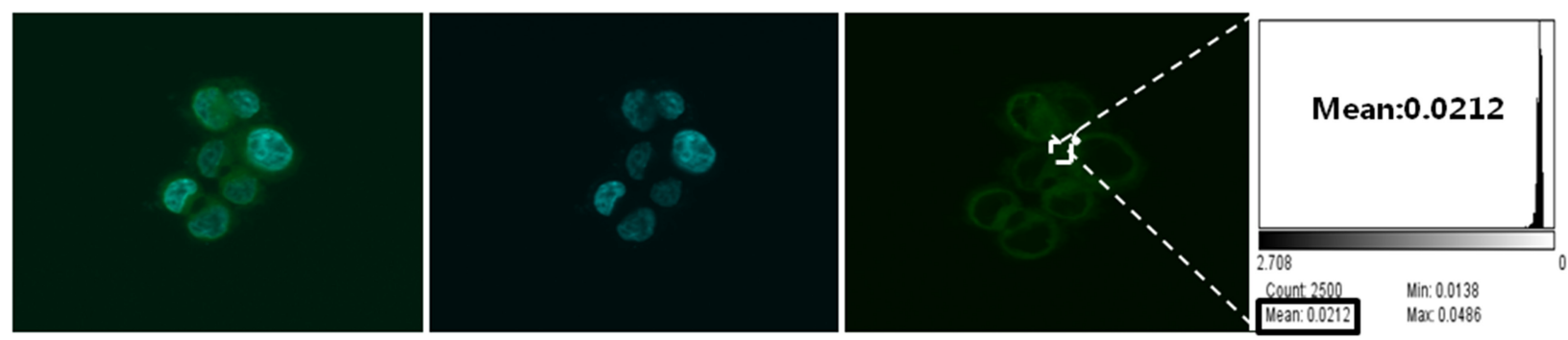

$\mathbf{F}$

\section{LOVO}
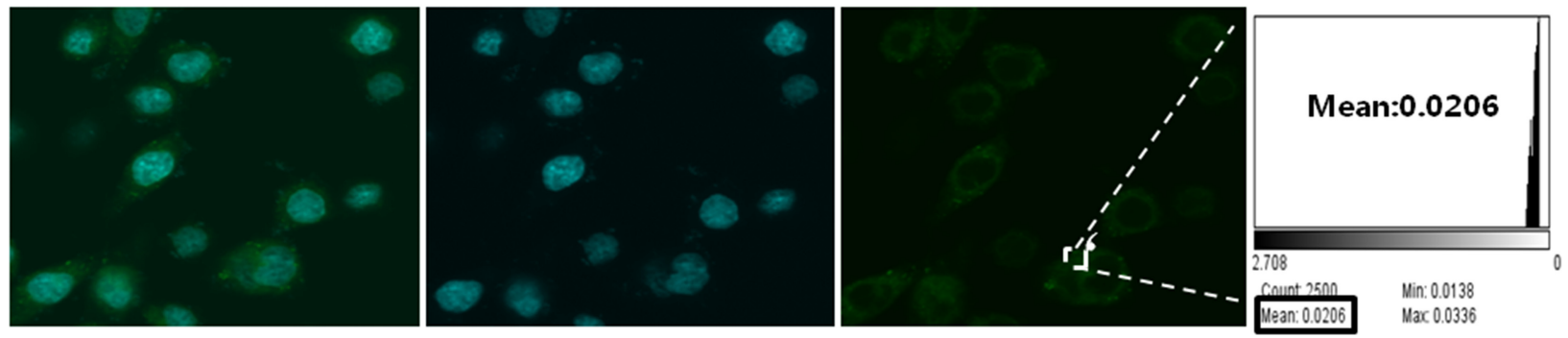

Figure 2: BODIPY-FA absorption by CRC cell lines and normal FHC cells. (A) Adherent cell uptake of BODIPY-FA at the indicated time points, as determined with FACS analysis. (B) BODIPY-FA uptake by suspensions of primary colonic normal and adenocarcinoma cells, as determined with FACS analysis. (C) The average fluorescence intensity of BODIPY-FA in colonic cells after CLSM screening. (D, E, F) CLSM imaging of adherent cell uptake of BODIPY-FA within 15 minutes, to confirm that FHC cells absorbed more BODIPY-FA than the CRC cell lines. $* P<0.05, * * P<0.01, * * * * P<0.0001$. Bars indicate the SEMs. 
images, 103 low-grade IN images, 132 high-grade IN images, and 124 adenocarcinoma images) of patients were analyzed under the same excitation brightness control. Images with obvious impurities on the tissue surface were eliminated from the assessment. The BODIPY-FA signals of non-neoplastic sites were almost 1.6-, 2- and 2.2-fold higher than those of low-grade IN, high-grade IN and cancerous sites, respectively, but the signals of high-grade IN and adenocarcinoma sites did not differ significantly from one another (Figure 6G).
A

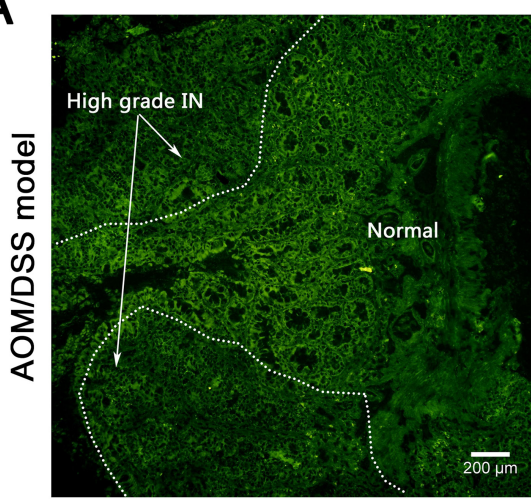

C

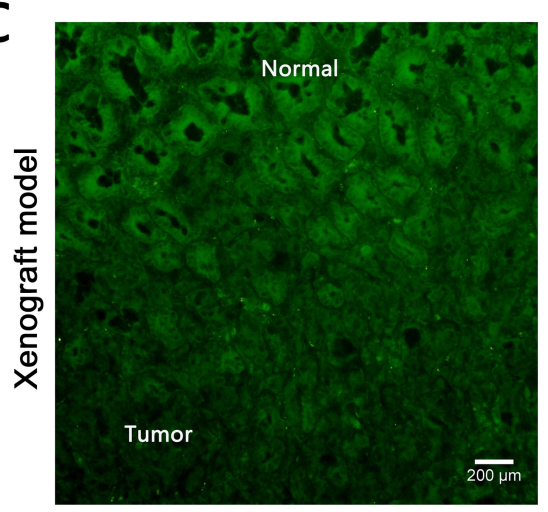

D

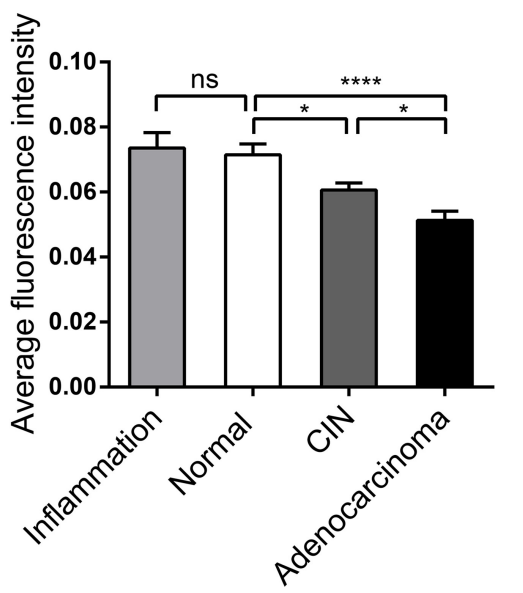

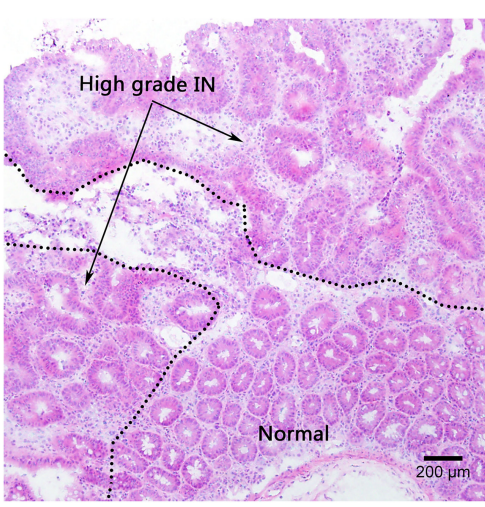

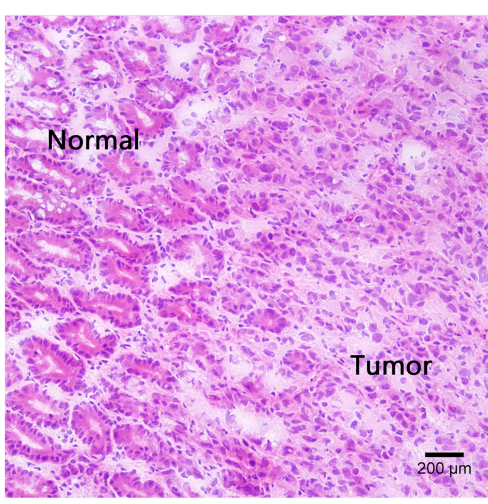

$\mathrm{E}$

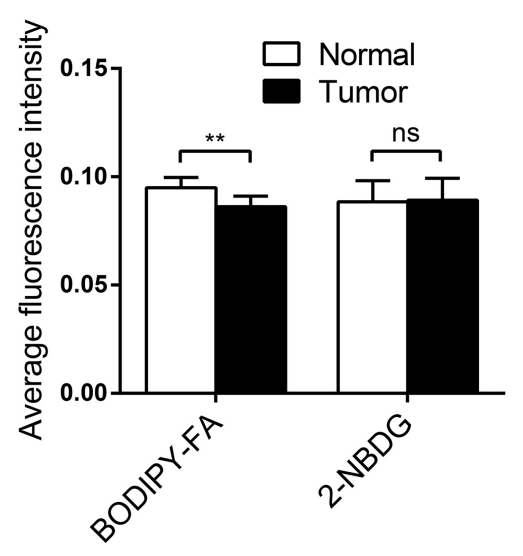

B
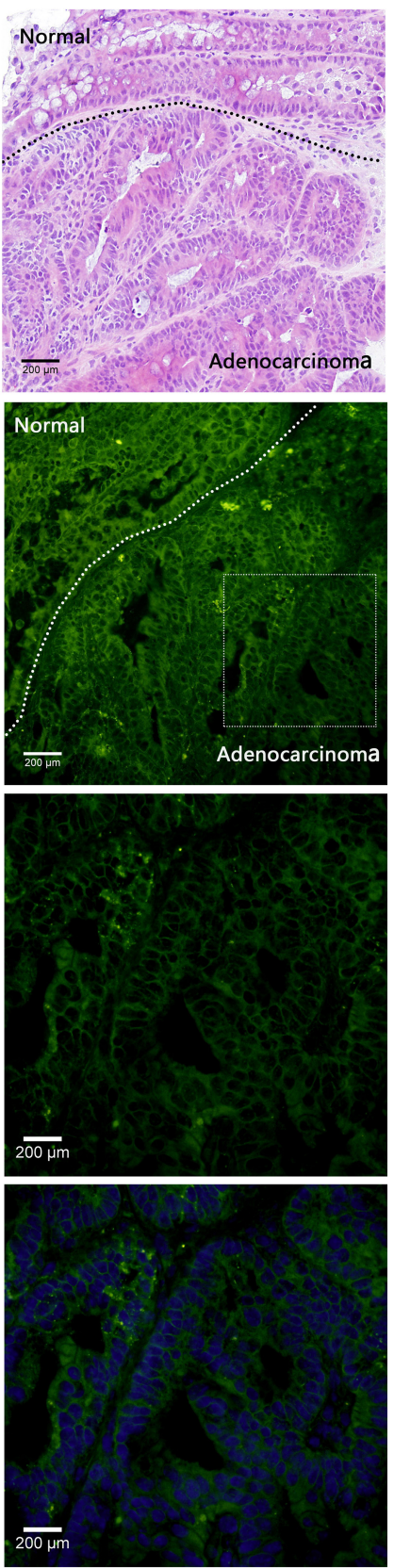

Figure 3: Fluorescence microscopy imaging after topical application of BODIPY-FA to the mucosae of mouse models, and the corresponding H\&E images. (A) BODIPY-FA signal at the transition between the normal mucosa and high-grade IN in an AOM/DSS-induced mouse at the end of three cycles. Images were obtained at 100×. (B) BODIPY-FA staining of the transition between normal and adenocarcinoma mucosae in an AOM/DSS-induced mouse, as observed at 200×; the details of the adenocarcinoma and the corresponding DAPI nuclear counterstaining are presented at 400×. (C) Imaging of a LOVO xenograft mouse at the transition between normal and tumorous tissue, four weeks after implantation, shown at 200×. (D) The average fluorescence intensity of BODIPY-FA in different tissue types of AOM/DSS-induced mice. (E) The average fluorescence intensities of BODIPY-FA and 2-NBDG in normal and tumorous tissues from xenograft mice. ${ }^{*} P<0.05,{ }^{*} P<0.01, * * * * P<0.0001$; ns, not significant. Scale bars: $200 \mu \mathrm{m}$. Bars indicate the SEMs. 


\section{Staining characteristics of BODIPY-FA and classification of colorectal neoplasms by CLE}

We also analyzed the characteristics of BODIPYFA staining with CLE. At normal sites, black nuclei on the basal side and bright cytoplasmic staining facilitated the visualization of regularly arranged crypts, which were surrounded by epithelial cells and goblet cells. With regard to hyperplastic polyps, focal aggregations of regularly shaped crypts or typical star-shaped luminal crypt openings were visible, and the number of goblet cells was normal or slightly reduced (Figure 6A, 6B). BODIPYFA staining on adenocarcinomas was greatly altered, revealing many atypical cells with large and irregularly shaped nuclei (Figure 6E, 6F).

Next, we evaluated the potential use of BODIPY-FA with CLE for the early diagnosis of neoplasms. In lowgrade IN, BODIPY-FA-stained lesions exhibited slightly atypical or elongated glands with a single layer of nuclei on the basal side. Moreover, the nucleus-to-cytoplasm ratio was almost normal, in spite of the slightly reduced number of goblet cells. Strikingly, for high-grade IN, ROIs

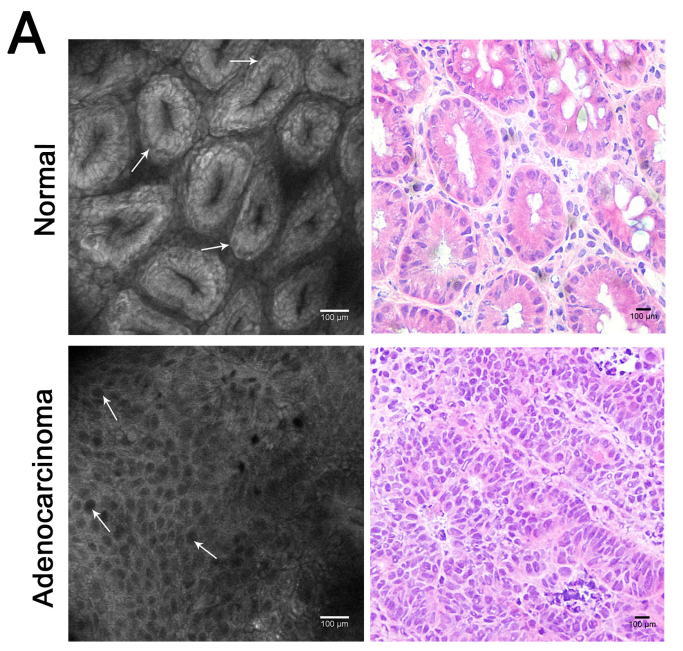

\section{B}

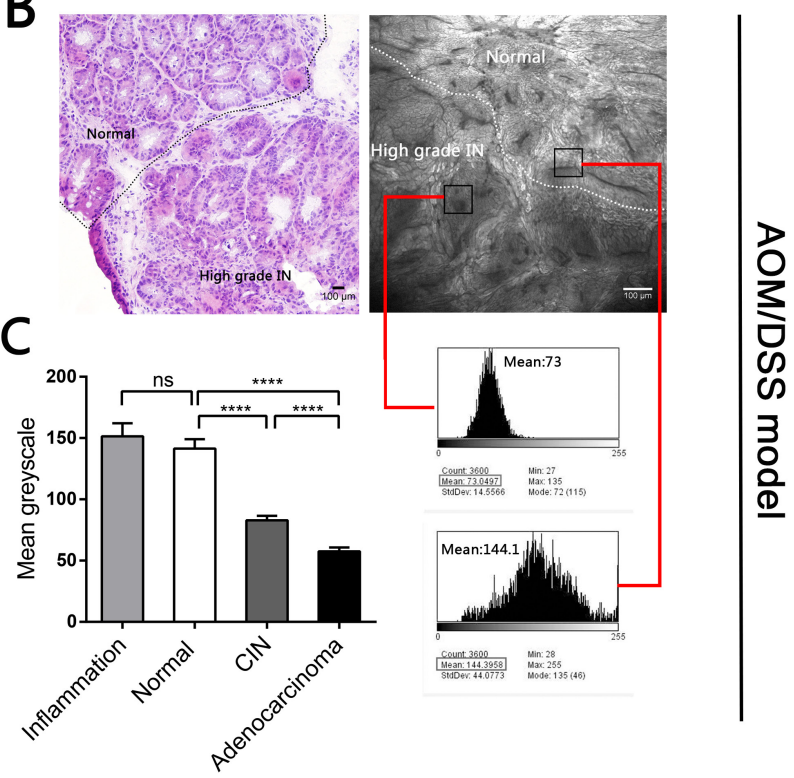

D

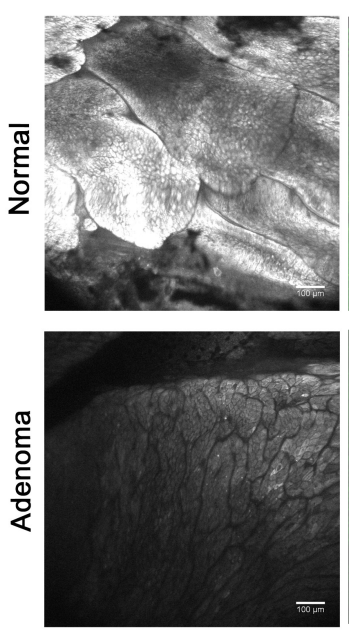

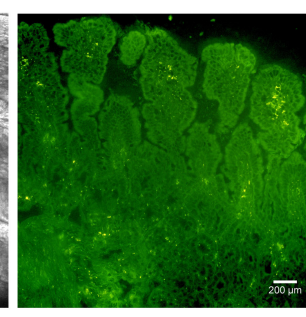

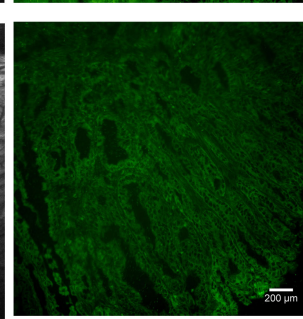

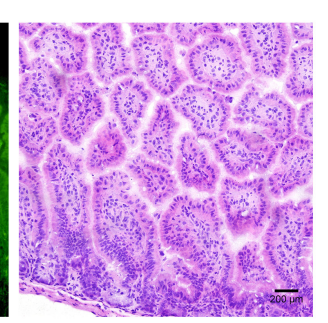

E
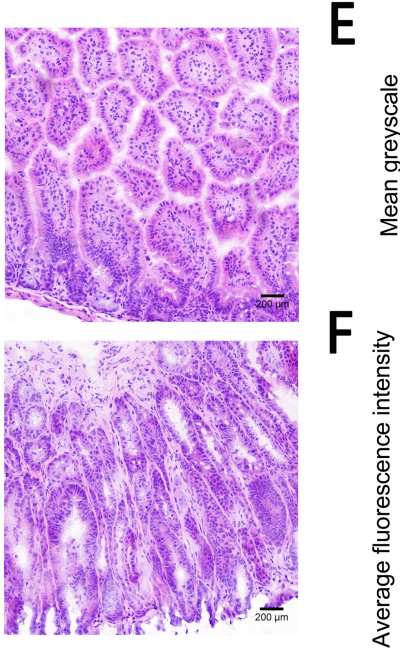

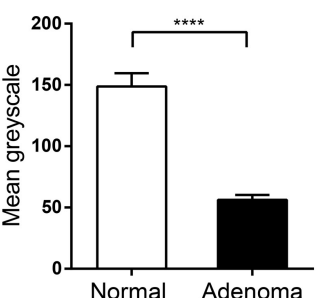

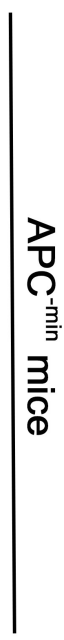

Figure 4: CLE images following topical application BODIPY-FA to the mucosae of mouse models, and the corresponding H\&E images. (A) BODIPY-FA expression in normal and adenocarcinoma mucosae from the AOM/DSS mouse model. Arrows indicate the morphology of the nuclei. (B) Imaging of BODIPY-FA at the transition between the normal mucosa and high-grade IN in an AOM/ DSS-induced mouse, and the corresponding H\&E images. Histograms display the greyscale values of the normal and high-grade IN regions. (C) The mean greyscale values of the BODIPY-FA signals in different tissue types of AOM/DSS-induced mice. (D) BODIPY-FA staining in normal and adenoma sites of $\mathrm{APC}^{-\mathrm{min}}$ mice, as observed with CLE, and the corresponding fluorescence imaging. (E) The mean greyscale values of the BODIPY-FA signals in APC-min mice. (F) The average intensity of BODIPY-FA in APC ${ }^{- \text {min }}$ mice. ${ }^{* * *} P<0.001$, $* * * * P<0.0001 ;$ ns, not significant. Bars indicate the SEMs. 
displayed lateral fusion of destroyed crypts, extremely elongated and branch-like crypts, and an increased nucleusto-cytoplasm ratio. In addition, goblet cells were either reduced in number or completely absent (Figure 6C, 6D).

\section{Comparison between CLE diagnosis and clinical histological findings}

Finally, we performed a clinical comparison of CLE diagnosis and histological assessments. The interobserver agreement was greater for BODIPY-FA staining $(\kappa=0.72, P<0.001)$ than for fluorescein sodium $(\kappa=0.61$, $P<0.001)$. The overall validity of CLE versus $\mathrm{H} \& \mathrm{E}$ diagnosis was $74.65 \%$ with BODIPY-FA and $55.88 \%$ with fluorescein sodium. In addition, there was good diagnostic consistency $(\kappa=0.68, P<0.001)$ between BODIPYFA images and H\&E diagnosis. However, although the diagnostic difference was slight (chi-square $=1.10$, $P=0.9540$ ), the consistency with the histological diagnosis was worse for fluorescein sodium than for BODIPYFA $(\kappa=0.43, P<0.001)$. These results are detailed in Supplementary Tables 2 and 3, indicating that CLE with BODIPY-FA staining had greater consistency and overall validity than CLE with intravenous fluorescein sodium.

\section{DISCUSSION}

Endoscopic treatment for CRC at the early stage can effectively improve the cure rate for patients. In this study, we found that lipid metabolism was aberrant in CRC, and that the uptake of the fluorescently labeled FA BODIPYFA was reduced in CRC tissues from both mouse models and human patients. BODIPY-FA staining under CLE effectively differentiated non-neoplastic tissues, colonic IN
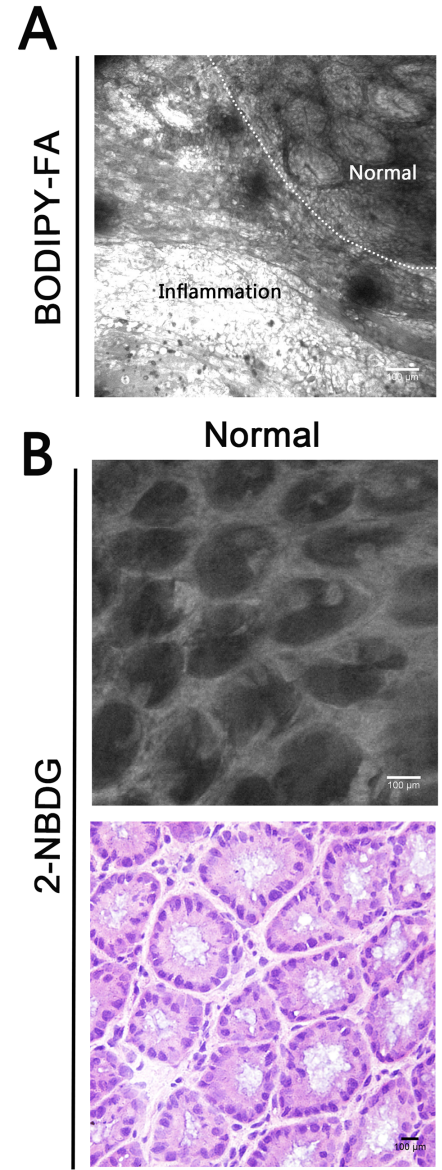

AOM/DSS model

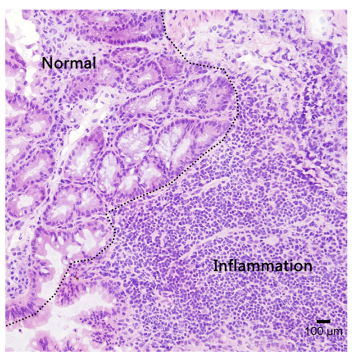

\section{Adenocarcinoma}
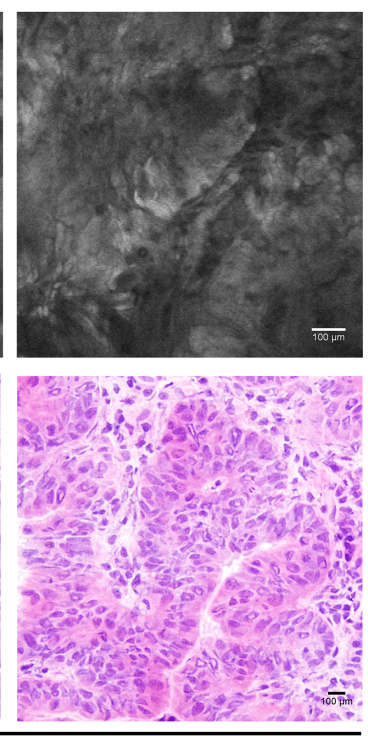

C
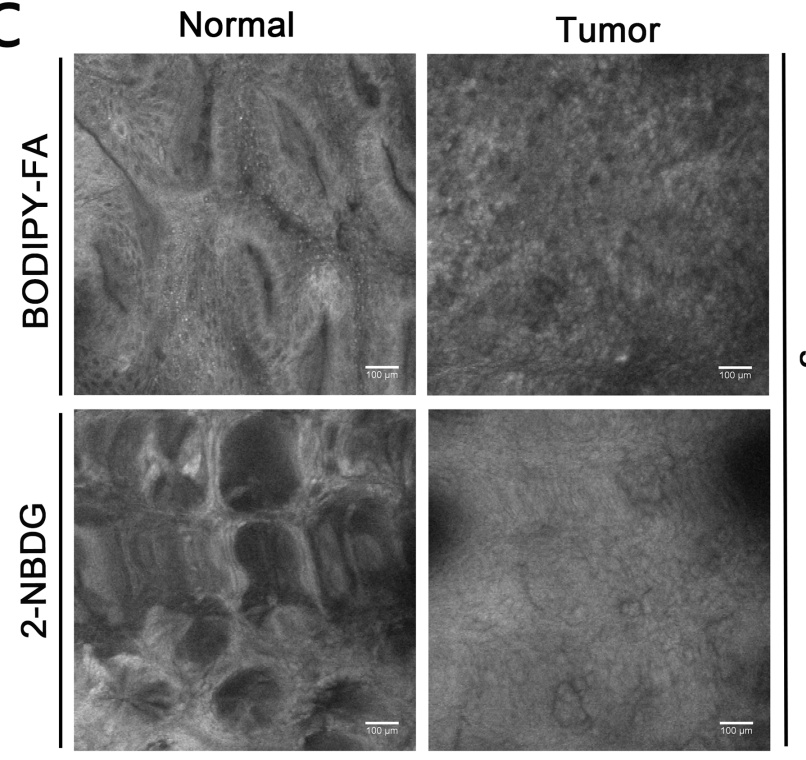

E
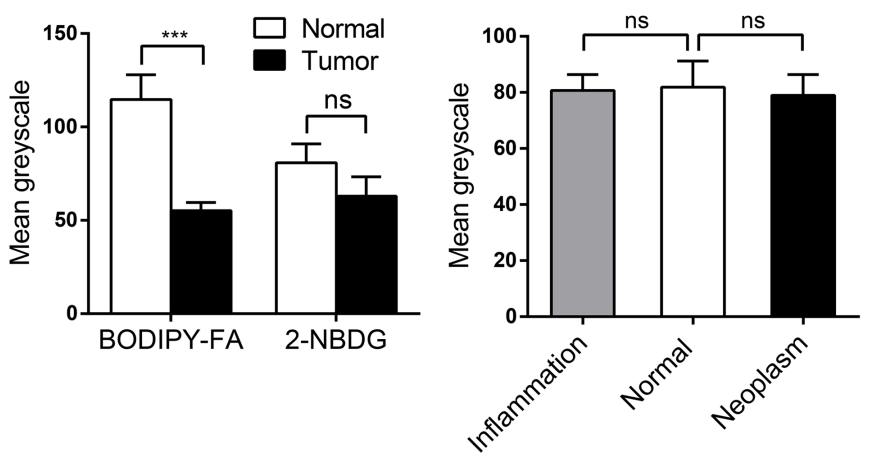

Figure 5: CLE images following topical application of fluorescent agents to the mucosae of mouse models, and corresponding H\&E images. (A) Imaging of BODIPY-FA signals at the transition between inflammation and the normal mucosa of an AOM/DSS-induced mouse at the end of one cycle. (B) 2-NBDG staining in normal and adenocarcinoma mucosae. (C) BODIPY-FA and 2-NBDG staining in normal and tumorous sites of LOVO xenograft mice. (D) The mean greyscale values of the fluorescent signals in normal and tumorous regions of LOVO xenograft mice. (E) The mean greyscale values of the 2-NBDG signals in different tissue types of AOM/DSS-induced mice. ${ }^{* * *} P<0.001$; ns, not significant. Bars indicate the SEMs. 
and carcinomas, as it created a strong fluorescent contrast and allowed clear visualization of cellular structures. BODIPY-FA with CLE enabled real-time cellular and sub-cellular imaging of ROIs, and defined a much more accurate boundary between neoplasm and non-neoplasm than existing methods; thus, this technique may facilitate early CRC identification for further clinical treatments.

Multiple novel endoscopic technologies have been investigated for early CRC identification, such as NBI,
FICE, MCE and CLE. NBI can differentiate neoplasms from non-neoplasms according to surface microvessel changes, but poorly detects depressed-type lesions [35]. MCE with crystal violet staining has a diagnostic accuracy of $85-88 \%$ based on surface pit pattern identification [36]. However, disappointing misdiagnoses and missed diagnoses still occur, due to the variety of superficial lesions and the limited role of forceps biopsy; in fact, the disagreement between histopathology and resected biopsy
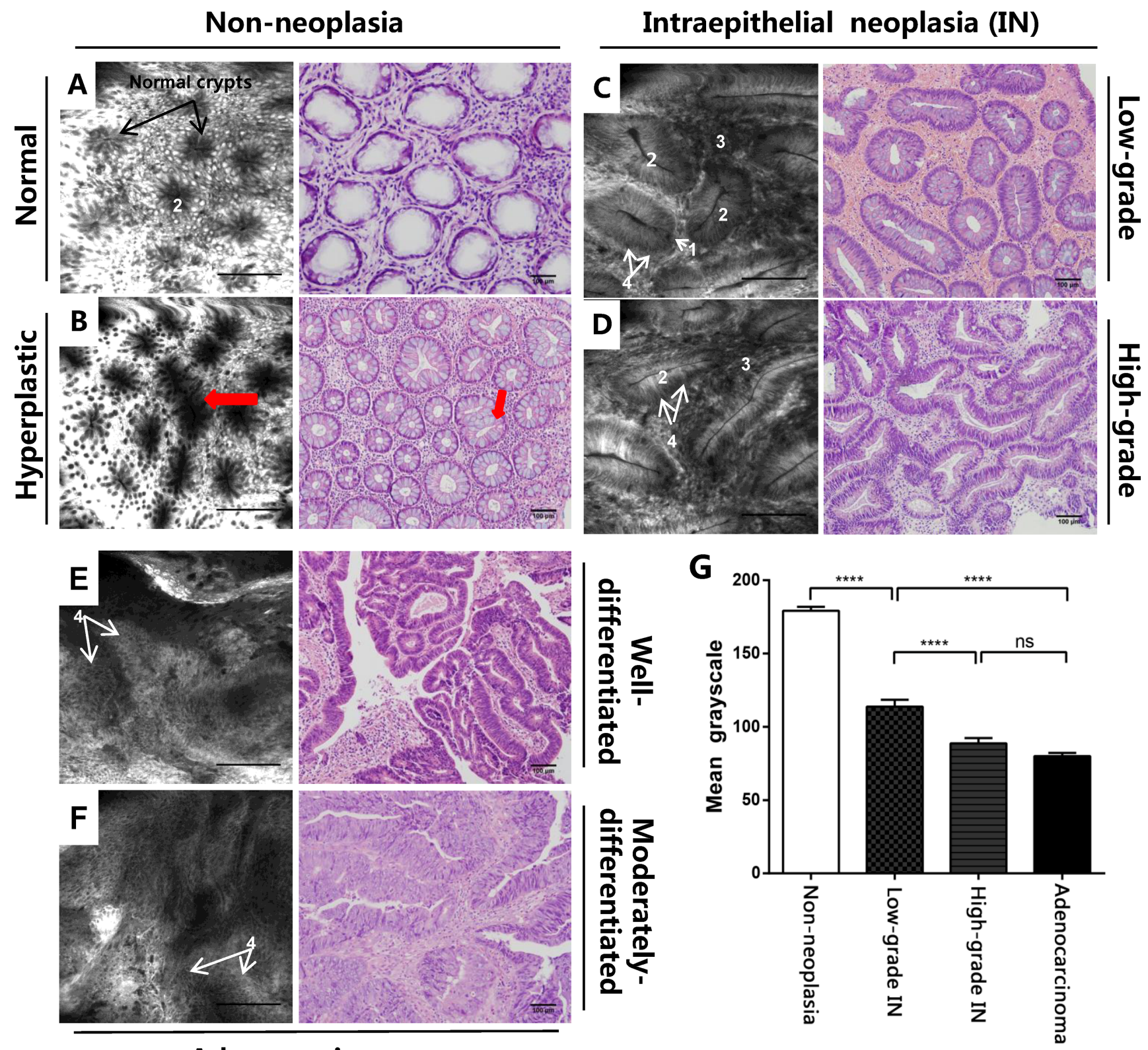

Adenocarcinoma

Figure 6: CLE imaging of patient samples and the corresponding H\&E images. (A) CLE imaging of the normal colonic mucosa and (B) typical star-shaped crypt openings and fused regularly shaped crypts (arrowheads) at the regenerative location. (C) Images of mucosal low-grade IN display elongated crypts with reduced goblet cells. (D) Images of high-grade IN display branch-like or extremely stretched crypts with lateral fusion of irregular glands and an elevated nucleus-to-cytoplasm ratio. (E) A well-differentiated adenocarcinoma exhibits enlarged black nuclei, with an enhanced nucleus-to-cytoplasm ratio. (F) Images of a moderately-differentiated adenocarcinoma exhibit extremely disorganized or absent glands. (G) The mean greyscale values of BODIPY-FA signals in different tissue types from patient samples. Legend: 1. Goblet cells, 2. Crypt lumen, 3. Lamina propria, 4. Nucleus. ****P<0.0001; ns, not significant. Bars indicate the SEMs. 
tissues has been reported to be approximately 40\% [37]. In contrast, with its $1000 \times$ magnifying visualization, CLE has been shown to have $93.9 \%$ sensitivity and $95.9 \%$ specificity for colonic adenoma detection [38].

CLE diagnosis has relied exclusively on fluorescent staining agents such as fluorescein sodium and acriflavine thus far. BODIPY-FA is a C18:0 analogue, and can distinctly highlight the structure of the cell nucleus in mouse models when it is applied topically. Although systemic agents such as fluorescein sodium have the advantage of reduced immunogenicity, their localization in blood vessels prevents them from displaying the morphology of the intracellular architecture. Topical agents have been used preferentially in clinical settings $[39,40]$, since they allow real-time imaging of ROIs and can be applied easily during CLE. In our trial, due to its low molecular weight and hydrophobic features, BODIPYFA rapidly entered the cytoplasm (within five minutes) and accumulated stably in the cytoplasm. Given its rapid and relatively stable imaging of ROIs, CLE screening with BODIPY-FA is more convenient and efficient than CLE with fluorescein sodium.

In addition to rapid cellular imaging, in our study, BODIPY-FA with CLE effectively visualized enlarged glands with different arrangements and, more importantly, distinguished between normal and aberrant nucleus-to-cytoplasm ratios in both mouse models and human tissues. In contrast, 2-NBDG failed to visualize nuclei or glands under CLE. Furthermore, the overall prediction consistency and accuracy were much greater for CLE with BODIPY-FA than with fluorescein sodium. Thus, malignant lesions displayed weak BODIPY-FA signals, accompanied by increased nucleus-to-cytoplasm ratios under CLE. In addition, as the elevated BODIPYFA signals in inflamed sites were parallel to those in normal mucosae, BODIPY-FA imaging with CLE also facilitated the detection of colitis-associated carcinoma, thus assisting in the diagnosis of endoscopic depressedtype lesions. Overall, BODIPY-FA staining under CLE exploited differences in lipid metabolism and enhanced cellular visualization, thereby improving the diagnostic efficiency of CLE screening during CRC identification.

De novo FA synthesis is a common metabolic characteristic of cancer, and has been studied in various human cancers, including breast, colorectal, lung, prostate and liver cancer $[41,42]$. This process is controlled by ratelimiting enzymes such as FASN and ACC, and generates FAs de novo from acetyl-CoA. Inhibitors of FASN (C75 or TVB-3166) or ACC (ND-646) have been shown to effectively suppress the growth and viability of cancer cells, demonstrating the importance of endogenous FA synthesis in oncology [43, 44]. Strikingly, exogenous FAs, in addition to being the main source of cellular energy, generally compete with de novo FA synthesis [45, 46]. In a previous study, growing cells in lipid-depleted medium clearly enhanced the effects of ND-646 by placing a heavier burden on endogenous FA synthesis [44]. On the other hand, the addition of palmitate fully restored cancer cell viability after the inhibition of FASN [43]. In our study, we found that FASN expression was increased in human CRC, while the expression of membranal transporters of LCFAs such as FABP1, CD36 and Caveolin-1 was reduced. The reduced expression of lipid transporters may further impair extracellular lipid uptake in CRC tissues relative to normal colonic mucosae. Further BODIPY-FA detection in vitro and in vivo also verified these findings. Thus, we propose that limited exogenous lipid usage directly manifests itself through the downregulation of LCFA transporters, and could be a response to increased de novo FA synthesis in CRC tissues. Further molecular research could focus on this interesting subject.

In summary, abnormal lipid metabolism in cancer cells caused distinct uptake patterns of the fluorescently labeled FA BODIPY-FA in non-neoplastic mucosae and neoplastic tissues, providing a novel approach for early CRC detection. This method specifically identified malignant sites with "dark areas" and allowed welldefined intracellular imaging under CLE, similar to "real-time histochemistry," which enhanced the detection of suspicious lesions and enabled relatively reliable diagnoses. This method was also beneficial for the detection of inflammatory bowel disease-associated carcinoma, and could be a reliable screening tool for atrisk patients. We speculate that reduced exogenous FA uptake may be an inherent neoplastic characteristic that is representative of most other tumors. This research will open a new door to tracing pre-neoplastic or neoplastic lesions in scientific research and clinical practice.

\section{MATERIALS AND METHODS}

\section{Cell culture and fluorescence-activated cell sorting (FACS)-based BODIPY-FA uptake assays}

All cells were obtained from the American Type Culture Collection (Maryland, USA). Different CRC cell lines were cultured in Dulbecco's Modified Eagle Medium (10\% fetal calf serum, $1 \%$ glutamine, $1 \%$ streptomycin /penicillin) at $37^{\circ} \mathrm{C}$ and $5 \% \mathrm{CO}_{2}$. When cells reached $60-80 \%$ confluency, they were plated in 12 -well plates covered with aluminum foil at a density of 500,000 cells/ well and incubated overnight for adherence. BODIPY-FA (D3823, Invitrogen, Carlsbad, CA, USA) was dissolved in dimethyl sulfoxide as a stock solution [25], and the working solution was diluted with $1 \times$ phosphate-buffered saline (PBS) and $0.1 \%$ FA-free bovine serum albumin. Different cell lines were cultivated in $2 \mu \mathrm{M}$ BODIPYFA for 1-15 minutes. For fluorescent competition with stearic acid, SW480 cells were cultivated in various concentrations of a BODIPY-FA-stearic acid mixture for 15 minutes. Then, the cells were rinsed and harvested immediately for FACS analysis. 
We also isolated primary colonic epithelial cells from cancerous and matched normal tissue samples, as described previously [26], after obtaining informed consent from the patients. Details on the patients are given in Supplementary Table 1.3.

For cellular staining with BODIPY-FA, FHC and CRC cell lines were cultured in confocal dishes and incubated with a 2- $\mu \mathrm{M}$ BODIPY-FA mix for 15 minutes. Cells were then fixed with $4 \%$ paraformaldehyde for 30 minutes, and the nuclei were counterstained with 4',6-diamidino-2-phenylindole (DAPI; 4083, Cell Signaling Technology, Boston, MA, USA). Following fixation, cells were washed three times with pre-chilled PBS. Images were acquired with an FV1000 confocal laser scanning microscope (CLSM).

\section{Animal model construction}

Three different mouse models of CRC were studied. The experiments were approved by the Animal Ethics Committee of Southern Medical University (L2015006) and conducted in accordance with the animal experimentation guidelines of the Chinese Ministry of Health. For the generation of the chemically induced mouse model, six- to eight-week-old male Balb/c mice were intraperitoneally injected with $10 \mathrm{mg} / \mathrm{kg}$ body weight azoxymethane (AOM; A5486, Sigma-Aldrich, Saint Louis, MO, USA). One week later, 2.5\% dextran sulfate sodium (DSS; MW 40000-50000 Da, MP, Santa Ana, California, USA) was dissolved in distilled water and administered for seven days, followed by 14 days of normal drinking water [27]. This cycle was repeated three times, and when the last DSS cycle ended, the mice were prepared for in vivo staining. Our run-in phase experiments demonstrated that the neoplastic regions always occurred near the rectum, with increased thickness and stiffness of the bowel wall macroscopically; these observations guided the topical application of the fluorescent agents. We successfully acquired 32 AOM/DSS-induced neoplastic mice; 14 were used for the first fluorescence microscopy imaging study (six for BODIPY-FA; six for 2-Deoxy-2[(7-nitro-2,1,3-benzoxadiazol-4-yl)amino]-D-glucose (2NBDG); and two for acriflavine hydrochloride), and 18 were used for in vivo CLE imaging (eight for BODIPY-FA; seven for 2-NBDG; and three for acriflavine hydrochloride).

For the xenograft model, $10^{6}$ human LOVO CRC cells were orthotopically implanted through a laparotomy into the colonic serosa layer of Balb/c null mice (Medical Experimental Animal Centre of Guangdong Province, China) under stringent aseptic conditions. Subsequently, the abdominal cavity was closed with a commonly used disinfectant. Three to four weeks after the implantation, the mice received a second laparotomy for the next step. In total, 16 xenograft mice were successfully acquired, including eight for fluorescence microscopy imaging and eight for in vivo CLE imaging (four for BODIPY-FA staining and four for 2-NBDG staining).
APC $^{-m i n}$ mice (purchased from the Animal Model Research Centre of Nanjing University, China) are a widely accepted model of intestinal tumorigenesis. Six $\mathrm{APC}^{-\mathrm{min}}$ mice (four to six months old) received follow-up BODIPY-FA imaging.

\section{Staining in the mouse models}

Prior to the application of fluorescent agents, AOM/ DSS-induced mice, xenograft mice and $\mathrm{APC}^{-\mathrm{min}}$ mice were fasted for $12 \mathrm{~h}$. Anesthesia was administered with $0.01 \mathrm{~g} / \mathrm{mL}$ pentobarbital, given as $6-7 \mu \mathrm{L} / \mathrm{g}$ body weight. For in vivo neoplastic staining, a $200 \mu \mathrm{M}$ BODIPY-FA mix, $500 \mu \mathrm{M}$ 2-NBDG (N13195, Invitrogen) and $0.05 \%$ acriflavine (A8251, Sigma-Aldrich) were prepared, and the colons and/or small intestines of the mice were isolated through laparotomy after a 2-3-cm long segment of the tract was created with two vascular hemoclips. Then, $100 \mu \mathrm{L}$ of the fluorescent agent was injected directly into the lumen. After five minutes of staining, the stained areas were exposed, and the unbound BODIPY-FA was rinsed off three times with PBS.

\section{Patients and tissue specimens}

The sporadic colorectal neoplasm patients came from the Departments of Gastroenterology and General Surgery, Nanfang Hospital, Guangzhou, China. These patients were selected based on the following factors: (1) complete clinical follow-up data were available, and (2) the patients had not eaten large amounts of high-fat food or been taking lipid medicine. The Institutional Research Medical Ethics Committee of Nanfang Hospital granted approval for the present study (NFEC-2015-083). Informed written consent was obtained from patients before examination.

\section{RT-PCR}

Total RNA was extracted with TRIzol (Takara) from paired normal and cancerous tissues from CRC patients, and cDNA was synthesized with TAKARA reverse transcriptase (037A, TAKARA, China) with oligo-dT primers. The cDNA and SYBR Green Premix ExTaq were used for subsequent real-time PCR amplification on an LC480 System. The primer sequences are listed in Supplementary Table 5.

\section{Western blotting}

The protein levels of fatty acid synthase (FASN), fatty acid binding protein 1 (FABP1), Caveolin-1 and CD36 in the paired human CRC samples were examined by Western blotting. Antibodies for FASN, FABP1, Caveolin-1, CD36 and GAPDH were purchased from Cell Signaling Technology. Protein blots were incubated with the indicated primary antibodies and then with the 
appropriate secondary antibodies, and detection was performed with enhanced chemiluminescence according to the manufacturer's instructions.

\section{Histochemistry}

Active specimens from each region of the mouse models were immediately embedded with Optimum Cutting Temperature compound in liquid nitrogen for cryosectioning after the staining procedure. After being counterstained with DAPI, the cryosections were observed with fluorescence microscopy. The other cryosections were stained with H\&E. Finally, the fluorescence images were matched with their corresponding H\&E images. The mean fluorescence intensity was calculated for statistical analysis. H\&E images were analyzed independently by B.N. and H.Z.

\section{CLE system}

CLE (EC3830FK, Pentax, Japan) integrates experimental confocal microscopy with conventional optical endoscopy, delivering an excitation wavelength of $488 \mathrm{~nm}$ and an emission wavelength of 505-585 nm. A confocal probe was placed onto the mucosal layer for scanning, and optical sections of $475 \times 475 \mu \mathrm{m}$ were obtained. The axial resolution was $7 \mu \mathrm{m}$, and the lateral resolution was $0.7 \mu \mathrm{m}(1024 \times 1024$ pixels $)$. The imaging depth (z-axis) varied from $0-250 \mu \mathrm{m}$ below the surface layer in $4-\mu \mathrm{m}$ increments. Confocal images were obtained at a fixed laser power of $500 \mu \mathrm{W}$ and standardized brightness.

\section{Staining of human tissues and CLE imaging}

Patients were randomly divided into the fluorescein sodium group and the BODIPY-FA group (detailed in Supplementary Tables 1.1 and 1.2). Fluorescein sodium (5-10 $\mathrm{mL}$ of a $10 \%$ solution, Baiyunshan Mingxing Pharmaceutical Co.) was administered intravenously according to a published protocol [28]. The 200- $\mu \mathrm{M}$ BODIPY-FA mix was applied topically for five minutes and then rinsed off with PBS.

For CLE imaging, when the confocal window is fully placed on the surface mucosa (for both in vivo mouse models and human tissues) and the imaging plane depth is real-time adjusted during ongoing screening, the observation time for each site should be shorter than 20 s. CLE was performed by two experienced endomicroscopists (W.G. or T.L.).

\section{Confocal pattern classification}

For the assessment of confocal images, clear images of each site were selected and evaluated according to previous classifications [20,29] by two different endoscopy doctors, with three main histopathologic characteristics taken into consideration. Disagreements between the two observers were resolved by discussion, and the final diagnosis was agreed upon by both physicians. One characteristic was crypt morphology, which allowed the images to be classified into normal mucosa, hyperplastic locations and neoplasia (intraepithelial neoplasia [IN] and adenocarcinoma). The second characteristic was nuclear visualization, which permitted differentiation among lowgrade IN, high-grade IN and adenocarcinoma. The last characteristic was the apparent darkness of the neoplasm compared with normal areas of the same patient under the same conditions. A detailed classification of the BODIPYFA images from our patient samples is shown in Table 1. The H\&E biopsy images were judged independently by two experts and graded according to the modified Vienna classification [30]. In addition, clear images with intravenous fluorescein sodium were selected and judged in strict accordance with previous classification criteria [20].

\section{Statistical analysis and evaluation}

Mean greyscale values and mean fluorescence intensities were analyzed with Image $\mathrm{J}(\mathrm{NIH}$, Bethesda, MD, USA). CLE images were analyzed based on the following standards: (1) the observation time was less than $20 \mathrm{~s}$ per site and (2) recognizable cellular structures were visible. ROIs of $100 \times 100 \mu \mathrm{m}$ from three different regions per image were selected for the calculation of the mean greyscale value and mean fluorescence intensity. Data were analyzed with the statistical software package SPSS v13.0 (SPSS Inc., Chicago, IL, USA). Significance was determined with Student's $t$-test, one-way or twoway ANOVA, or a nonparametric test, as appropriate. The Kolmogorov-Smirnov test was performed to compare relative protein expression in the primary data of 95 tumor samples from the Clinical Proteomic Tumor Analysis Consortium. All $P$ values were generated with two-sided tests, and $P<0.05$ was defined as significant.

\section{Double-blind trial for CLE diagnosis}

Seventy-one target locations from 17 patients, including up to 508 confocal images (mean: 7 per site, range: 5-20) with BODIPY-FA, and an additional 68 target locations from 45 patients, including up to 950 images (mean: 14 per site, range: 7-65) with fluorescein sodium, were randomly coded and separately sent to two expert endomicroscopists (FC.Z. and F.H.D.) who were blinded to the macroscopic appearance and H\&E diagnosis. All data were analyzed with SAS 9.4 software. The overall validity was calculated to compare the predictive ability of confocal endomicroscopy with that of the gold-standard H\&E diagnosis. The Bhapkar test and Kappa coefficient were used to evaluate the diagnostic difference and consistency, respectively, between H\&E staining and CLE with either BODIPY-FA or fluorescein sodium [31]. 
Table 1: Confocal laser endomicroscopy criteria for BODIPY-FA imaging for colorectal pathology

\begin{tabular}{|l|l|}
\hline Grade & Criteria \\
\hline Normal & Round luminal openings \\
Regular arrangement and distribution of crypts \\
A homogeneous layer of epithelial cells with normal polarity \\
Interspersed goblet cells \\
Approximately 10-15 crypts per field of view
\end{tabular}

The Kappa coefficient was also used to evaluate the interobserver agreement of the CLE diagnoses with BODIPYFA or fluorescein sodium staining.

\section{Writing assistance}

We acknowledge Dr. Zhifei Shao at Stanford University for his help in editing this manuscript.

\section{Authors' contributions}

FH.D., Y.F.: draft of the manuscript, data acquisition and analysis. W.G., T.L.: endoscopic operation. FC.Z., FH.D.: judgment of the confocal images. WL.Z., T.W.: proteomic analysis. J.W.: ethical application. ZY.S., XJ.Z.: material and technological support. B.N., H.Z.: judgment of the histopathology images. B.N.: study concept and design, acquisition of funding and study supervision.

\section{CONFLICTS OF INTEREST}

The authors have applied for an international patent about this study.

\section{FUNDING}

This study was sponsored by the Returned Scientific Research Foundation of the Ministry of Education and the National Natural and Science Foundation of China [81471080] to B.N. This work was also supported by the National Basic Research Program "973" of China [2014CBA0200203 to Bo Jiang1.] The 95 samples of protein data in this study were generated from the Clinical Proteomic Tumor Analysis Consortium Data Coordinating Center.

\section{REFERENCES}

1. DeBerardinis RJ. Is cancer a disease of abnormal cellular metabolism? New angles on an old idea. Genet Med. 2008; 10:767-777.

2. Kroemer G, Pouyssegur J. Tumor cell metabolism: cancer's Achilles' heel. Cancer Cell. 2008; 13:472-482.

3. Vander Heiden MG. Targeting cancer metabolism: a therapeutic window opens. Nat Rev Drug Discov. 2011; 10:671-684.

4. Vander Heiden MG, Cantley LC, Thompson CB. Understanding the Warburg effect: the metabolic 
requirements of cell proliferation. Science. 2009; 324:1029-1033.

5. Warburg O, Wind F, Negelein E. The metabolism of tumors in the body. J Gen Physiol. 1927; 8:519-530.

6. Mankoff DA, Eary JF, Link JM, Muzi M, Rajendran JG, Spence AM, Krohn KA. Tumor-specific positron emission tomography imaging in patients: $[18 \mathrm{~F}]$ fluorodeoxyglucose and beyond. Clin Cancer Res. 2007; 13:3460-3469.

7. Schoder H, Gonen M. Screening for cancer with PET, PET/ CT: potential and limitations. J Nucl Med. 2007; 48:4S-18S.

8. Zaidi N, Lupien L, Kuemmerle NB, Kinlaw WB, Swinnen JV, Smans K. Lipogenesis and lipolysis: the pathways exploited by the cancer cells to acquire fatty acids. Prog Lipid Res. 2013; 52:585-589.

9. Fritz V, Fajas L. Metabolism and proliferation share common regulatory pathways in cancer cells. Oncogene. 2010; 29:4369-4377.

10. Menendez JA, Lupu R. Fatty acid synthase and the lipogenic phenotype in cancer pathogenesis. Nat Rev Cancer. 2007; 7:763-777.

11. Zhang F, Du G. Dysregulated lipid metabolism in cancer. World J Biol Chem. 2012; 3:167-174.

12. Bhatt AP, Jacobs SR, Freemerman AJ, Makowski L, Rathmell JC, Dittmer DP, Damania B. Dysregulation of fatty acid synthesis and glycolysis in non-Hodgkin lymphoma. Proc Natl Acad Sci USA. 2012; 109:11818-11823.

13. Kuhajda FP, Jenner K, Wood FD, Hennigar RA, Jacobs LB, Dick JD, Pasternack GR. Fatty acid synthesis: a potential selective target for antineoplastic therapy. Proc Natl Acad Sci USA. 1994; 91:6379-6383.

14. Rashid A, Pizer ES, Moga M, Milgraum LZ, Zahurak M, Pasternack GR, Kuhajda FP, Hamilton SR. Elevated expression of fatty acid synthase and fatty acid synthetic activity in colorectal neoplasia. Am J Pathol. 1997; 150:201-208.

15. Medes G, Thomas A, Weinhouse S. Metabolism of neoplastic tissue. IV. A study of lipid synthesis in neoplastic tissue slices in vitro. Cancer Res. 1953; 13:27-29.

16. Jemal A, Bray F, Center MM, Ferlay J, Ward E, Forman D. Global cancer statistics. CA Cancer J Clin. 2011; 61:69-90.

17. Wanders LK, East JE, Uitentuis SE, Leeflang MM, Dekker E. Diagnostic performance of narrowed spectrum endoscopy, autofluorescence imaging, and confocal laser endomicroscopy for optical diagnosis of colonic polyps: a meta-analysis. The Lancet Oncology. 2013; 14:1337-1347.

18. Xu JF, Yang L, Jin P, Sheng JQ. Endoscopic Approach for Superficial Colorectal Neoplasms. Gastrointestinal tumors. 2016; 3:69-80.

19. Dunbar KB. Endomicroscopy in Barrett's esophagus. Gastrointest Endosc Clin N Am. 2013; 23:565-579.

20. Kiesslich R, Burg J, Vieth M, Gnaendiger J, Enders M, Delaney P, Polglase A, McLaren W, Janell D, Thomas S, Nafe B, Galle PR, Neurath MF. Confocal laser endoscopy for diagnosing intraepithelial neoplasias and colorectal cancer in vivo. Gastroenterology. 2004; 127:706-713.

21. Liu J, Zuo X, Li C, Yu T, Gu X, Zhou C, Li Z, Goetz M, Kiesslich R, Li Y. In vivo molecular imaging of epidermal growth factor receptor in patients with colorectal neoplasia using confocal laser endomicroscopy. Cancer Lett. 2013; 330:200-207.

22. Foersch S, Kiesslich R, Waldner MJ, Delaney P, Galle PR, Neurath MF, Goetz M. Molecular imaging of VEGF in gastrointestinal cancer in vivo using confocal laser endomicroscopy. Gut. 2010; 59:1046-1055.

23. Goetz M, Ziebart A, Foersch S, Vieth M, Waldner MJ, Delaney P, Galle PR, Neurath MF, Kiesslich R. In vivo molecular imaging of colorectal cancer with confocal endomicroscopy by targeting epidermal growth factor receptor. Gastroenterology. 2010; 138:435-446.

24. Hsiung PL, Hardy J, Friedland S, Soetikno R, Du CB, Wu AP, Sahbaie P, Crawford JM, Lowe AW, Contag CH, Wang TD. Detection of colonic dysplasia in vivo using a targeted heptapeptide and confocal microendoscopy. Nat Med. 2008; 14:454-458.

25. Nie B, Park HM, Kazantzis M, Lin M, Henkin A, Ng S, Song SJ, Chen YL, Tran H, Lai RB, Her C, Maher JJ, Forman BM, et al. Specific bile acids inhibit hepatic fatty acid uptake in mice. Hepatology. 2012; 56:1300-1310.

26. Oikonomou E, Kothonidis K, Taoufik E, Probert E, Zografos G, Nasioulas G, Andera L, Pintzas A. Newly established tumourigenic primary human colon cancer cell lines are sensitive to TRAIL-induced apoptosis in vitro and in vivo. Br J Cancer. 2007; 97:73-84.

27. Thaker AI, Shaker A, Rao MS, Ciorba MA. Modeling colitis-associated cancer with azoxymethane (AOM) and dextran sulfate sodium (DSS). J Vis Exp. 2012.

28. Gheonea DI, Saftoiu A, Ciurea T, Popescu C, Georgescu $\mathrm{CV}$, Malos A. Confocal laser endomicroscopy of the colon. J Gastrointestin Liver Dis. 2010; 19:207-211.

29. Goetz M, Toermer T, Vieth M, Dunbar K, Hoffman A, Galle PR, Neurath MF, Delaney P, Kiesslich R. Simultaneous confocal laser endomicroscopy and chromoendoscopy with topical cresyl violet. Gastrointest Endosc. 2009; 70:959-968.

30. Schlemper RJ, Riddell RH, Kato Y, Borchard F, Cooper HS, Dawsey SM, Dixon MF, Fenoglio-Preiser CM, Flejou JF, Geboes K, Hattori T, Hirota T, Itabashi M, et al. The Vienna classification of gastrointestinal epithelial neoplasia. Gut. 2000; 47:251-255.

31. Maxwell AE. Comparing the classification of subjects by two independent judges. Br J Psychiatry. 1970; 116:651-655.

32. Brass E, Hanson E, O'Tierney-Ginn PF. Placental oleic acid uptake is lower in male offspring of obese women. Placenta. 2013; 34:503-509.

33. Kolahi K, Louey S, Varlamov O, Thornburg K. RealTime Tracking of BODIPY-FA Long-Chain Fatty Acid in Human Term Placenta Reveals Unique Lipid Dynamics in Cytotrophoblast Cells. PLoS One. 2016; 11:e0153522. 
34. Bionaz M, Thering BJ, Loor JJ. Fine metabolic regulation in ruminants via nutrient-gene interactions: saturated longchain fatty acids increase expression of genes involved in lipid metabolism and immune response partly through PPAR- $\alpha$ activation. Br J Nutr. 2011; 107:179-191.

35. Misawa M, Kudo SE, Wada Y, Nakamura H, Toyoshima N, Hayashi S, Mori Y, Kudo T, Hayashi T, Wakamura K, Miyachi H, Yamamura F, Hamatani S. Magnifying narrowband imaging of surface patterns for diagnosing colorectal cancer. Oncol Rep. 2013; 30:350-356.

36. Choi HJ, Lee BI, Choi H, Choi KY, Kim SW, Song JY, Ji JS, Kim BW. Diagnostic accuracy and interobserver agreement in predicting the submucosal invasion of colorectal tumors using gross findings, pit patterns, and microvasculatures. Clinical endoscopy. 2013; 46:168-171.

37. Sung HY, Cheung DY, Cho SH, Kim JI, Park SH, Han JY, Park GS, Kim JK, Chung IS. Polyps in the gastrointestinal tract: discrepancy between endoscopic forceps biopsies and resected specimens. Eur J Gastroenterol Hepatol. 2009; 21:190-195.

38. Xie XJ, Li CQ, Zuo XL, Yu T, Gu XM, Li Z, Ji R, Wang Q, Li YQ. Differentiation of colonic polyps by confocal laser endomicroscopy. Endoscopy. 2011; 43:87-93.

39. Coda S, Thillainayagam AV. State of the art in advanced endoscopic imaging for the detection and evaluation of dysplasia and early cancer of the gastrointestinal tract. Clin Exp Gastroenterol. 2014; 7:133-150.

40. Goetz M, Wang TD. Molecular imaging in gastrointestinal endoscopy. Gastroenterology. 2010; 138:828-833.e821.
41. Che L, Pilo MG, Cigliano A, Latte G, Simile MM, Ribback S, Dombrowski F, Evert M, Chen X, Calvisi DF. Oncogene dependent requirement of fatty acid synthase in hepatocellular carcinoma. Cell cycle. 2017; 16:499-507.

42. Li L, Che L, Tharp KM, Park HM, Pilo MG, Cao D, Cigliano A, Latte G, Xu Z, Ribback S, Dombrowski F, Evert $\mathrm{M}$, Gores GJ, et al. Differential requirement for de novo lipogenesis in cholangiocarcinoma and hepatocellular carcinoma of mice and humans. Hepatology. 2016; 63:1900-1913.

43. Ventura R, Mordec K, Waszczuk J, Wang Z, Lai J, Fridlib M, Buckley D, Kemble G, Heuer TS. Inhibition of de novo Palmitate Synthesis by Fatty Acid Synthase Induces Apoptosis in Tumor Cells by Remodeling Cell Membranes, Inhibiting Signaling Pathways, and Reprogramming Gene Expression. EBioMedicine. 2015; 2:808-824.

44. Svensson RU, Parker SJ, Eichner LJ, Kolar MJ, Wallace M, Brun SN, Lombardo PS, Van Nostrand JL, Hutchins A, Vera L, Gerken L, Greenwood J, Bhat S, et al. Inhibition of acetyl-CoA carboxylase suppresses fatty acid synthesis and tumor growth of non-small-cell lung cancer in preclinical models. Nature medicine. 2016; 22:1108-1119.

45. Rohrig F, Schulze A. The multifaceted roles of fatty acid synthesis in cancer. Nat Rev Cancer. 2016; 16:732-749.

46. Currie E, Schulze A, Zechner R, Walther TC, Farese RV Jr. Cellular fatty acid metabolism and cancer. Cell metabolism. 2013; 18:153-161. 